\title{
Numerical Simulations of Thermoelectric Materials for Local Seebeck Coefficient Measurements by a Heated Microprobe*
}

\author{
Chung-Chien HUANG** and Chen-I HUNG** \\ ** Department of Mechanical Engineering, National Cheng Kung University, \\ No.1, University Road, Tainan City 70101, Taiwan (R.O.C) \\ E-mail: n1891126@mail.ncku.edu.tw
}

\begin{abstract}
This paper presents numerical simulations of thermoelectric materials for local Seebeck coefficient measurements by a heated microprobe. The Galerkin finite element method is used to solve the governing equations with boundary conditions to obtain temperature and induced thermoelectric voltage distributions. The measured local Seebeck coefficients obtained from the potential Seebeck microprobe (PSM) model and the simplified PSM model are compared; the influence of probe tip size and heating time is investigated. A method is proposed for obtaining the spatial resolution of the PSM apparatus by increasing inhomogeneity size gradually. The simulation results indicate that the simplified PSM model can be efficiently used to simulate the local Seebeck coefficient measurements by the PSM apparatus. The temperature distribution is similar to the voltage distribution for homogeneous thermoelectric material but is different from the distorted voltage distribution for thermoelectric material with inhomogeneity. The spatial resolution of the PSM apparatus increases with decreasing probe tip size, heating time, and thermal conductivity of the thermoelectric material.
\end{abstract}

Keywords: Thermoelectric Material, Local Seebeck Coefficient, Measurement, Spatial Resolution, Inhomogeneity

\section{Introduction}

Thermoelectric materials can be used in power generators and refrigerators to convert heat directly into electricity when operating in the Seebeck mode or reversely in the Peltier mode $^{(1)}$. The performance of a thermoelectric material is often determined by the dimensionless figure of merit $\mathrm{ZT}=\alpha^{2} \sigma \mathrm{T} / \mathrm{k}^{(2)}$, where $\alpha$ is the Seebeck coefficient, $\sigma$ is the electric conductivity, $\mathrm{T}$ is the absolute temperature, and $\mathrm{k}$ is the thermal conductivity. Several methods can be used to improve the figure of merit, such as reducing the lattice thermal conductivity by increasing phonon interface scattering ${ }^{(3-4)}$ and fabricating functionally graded materials (FGM) by doping ${ }^{(5)}$. Therefore, the ability to directly measure the above material properties $(\alpha, \sigma$, and $\mathrm{k}$ ) is important for developing new thermoelectric materials with a high figure of merit.

When a thermoelectric material is subjected to a temperature difference, a potential difference is produced. The Seebeck coefficient $\alpha$ is defined as the ratio of the potential difference to the temperature difference under the open circuit condition. The Seebeck coefficient can be measured by the integral method or the differential method ${ }^{(6)}$. The integral method is often applied in high-temperature thermoelectric energy conversion and for samples with large dimensions ${ }^{(7)}$. The differential method is usually used to measure the

Received 2 Nov., 2010 (No. 10-0509)

DOI: 10.1299/jtst.6.178]

Copyright $\odot 2011$ by JSME 
Seebeck coefficient at high and low temperatures where there is a small temperature difference and for samples with small dimensions ${ }^{(7)}$. An apparatus called a potential Seebeck microprobe (PSM) can be used to measure the local Seebeck coefficients on macro and micro scales even for thermoelectric materials with relatively small inhomogeneity ${ }^{(8)}$. The profile of the local Seebeck coefficient measured by a scanning PSM can be used to judge whether microstructures and inhomogeneities exist in thermoelectric materials ${ }^{(9-10)}$. The PSM apparatus can be used to measure the Seebeck coefficient of thermoelectric thin films without damaging the sample ${ }^{(10)}$. Wu et $a l^{(11)}$ found that the spatial resolution limitation of the PSM apparatus is about 20-50 $\mu \mathrm{m}$ and depends on the thermal conductivity of the thermoelectric material; in the other words, the local Seebeck coefficient of inhomogeneity are not effective to be measured by the PSM apparatus when the dimensions of an inhomogeneity are smaller than $20 \mu \mathrm{m}$. The reported spatial resolution of the PSM apparatus is $10 \mu \mathrm{m}$, depending on the thermal conductivity of the thermoelectric sample ${ }^{(8-10)}$. However, it has not been shown how to obtain the spatial resolution limitation of the PSM using actual measurements. Many studies have proposed methods for measuring the Seebeck coefficient but few have reported detailed numerical simulations of local Seebeck coefficient measurements by the PSM apparatus. It is important to understand how inhomogeneity affects the measured Seebeck coefficient via the induced thermoelectric voltage contour and how the magnitude of the temperature difference zone affects the spatial resolution of the PSM.

The present study simulates local Seebeck coefficient measurements using the heated microprobe of the PSM apparatus. Three types of the thermoelectric material measured with various parameters (probe tip size and heating time) are simulated to obtain the local Seebeck coefficient using the PSM model and the simplified PSM model to determine which model is more accurate and efficient. The sample temperature distribution caused by heated microprobe contact is simulated to study the relationship between the probe tip size, heating time, and temperature difference zone. The induced thermoelectric voltage distribution is acquired to understand the influence of an inhomogeneity with a Seebeck coefficient that is different from the examined example on the examined sample and on the sample-tip contact area. The dependence of the spatial resolution of the PSM apparatus for measuring the local Seebeck coefficient on the probe tip size, heating time, and thermal conductivity of the examined material is also investigated.

\section{Nomenclature}

c :specific heat, $\mathrm{JK}^{-1} \mathrm{Kg}^{-1}$

$\mathrm{k}$ :thermal conductivity, $\mathrm{WK}^{-1} \mathrm{~m}^{-1}$

$\mathrm{t} \quad$ :time, $\mu \mathrm{s}$

$\mathrm{T}$ :temperature, $\mathrm{K}$

$\mathrm{V}$ :thermoelectric voltage, $\mathrm{V}$

$\mathrm{U}$ :thermoelectric voltage without considering the Seebeck coefficient of wires, $\mathrm{V}$

$\mathrm{U}_{1}$ :thermoelectric voltage measured between $\mathrm{Cu}-\mathrm{Cu}$ wires, $\mathrm{V}$

$\mathrm{U}_{2}$ :thermoelectric voltage measured between $\mathrm{CuNi}-\mathrm{CuNi}$ wires, $\mathrm{V}$

$\Delta \mathrm{T}$ :temperature difference, $\mathrm{K}$

$\Delta \mathrm{V}$ :thermoelectric voltage difference, $\mathrm{V}$

$\alpha \quad$ :Seebeck coefficient of the thermoelectric material, $\mu \mathrm{V} / \mathrm{K}$

$\alpha_{\mathrm{s}} \quad$ :Seebeck coefficient of sample, $\mu \mathrm{V} / \mathrm{K}$

$\alpha_{\mathrm{Cu}}$ :Seebeck coefficient of $\mathrm{Cu}, \mu \mathrm{V} / \mathrm{K}$

$\alpha_{\mathrm{CuNi}}$ :Seebeck coefficient of $\mathrm{CuNi}, \mu \mathrm{V} / \mathrm{K}$

$\rho \quad$ :density, $\mathrm{Kg} \cdot \mathrm{m}^{-3}$ 


\section{Numerical Simulations}

\subsection{Principle of PSM apparatus}

When a thermocouple (as shown in Fig. 1) formed from two dissimilar conductors (A and $\mathrm{B}$ ) is subjected to a small temperature difference $(\Delta \mathrm{T})$ between two junctions ( $\mathrm{C}$ and $\mathrm{D})$, an open-circuit potential difference $(\Delta \mathrm{V})$ is induced between the two B segments. The Seebeck coefficient $\alpha_{\mathrm{AB}}$ is defined as ${ }^{(12)}$ :

$$
\alpha_{\mathrm{AB}}=\alpha_{\mathrm{A}}-\alpha_{\mathrm{B}}=\frac{\Delta \mathrm{V}}{\Delta \mathrm{T}}
$$

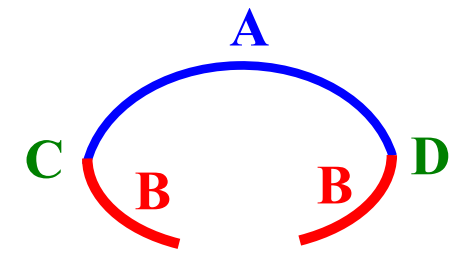

Fig. 1 Schematic of a basic thermocouple.

In this study, the PSM apparatus ${ }^{(8-11,13-14)}$, which comprises a heated microprobe and two thermocouples, is used to measure the local Seebeck coefficient of a sample (as shown in Fig. 2). The temperature of the heated probe is maintained at $T_{1}$. The initial temperature of the sample keeps at $\mathrm{T}_{0}$. One thermocouple is placed in the probe to measure the probe temperature and the other is placed on the sample to measure the initial sample temperature. The difference between $T_{1}$ and $T_{0}$ is about $5 \mathrm{~K}$. When a heated probe tip is placed on the surface of the sample for a short time, the transient heat transfer in the sample induces thermoelectric voltages $\mathrm{U}_{1}$ and $\mathrm{U}_{2}$, which are measured simultaneously through $\mathrm{Cu}-\mathrm{Cu}$ and CuNi-CuNi wires, respectively. The induced thermoelectric voltages $U_{1}$ and $U_{2}$ can be written as follows ${ }^{(8-11,13-14)}$ :

$$
\begin{aligned}
& \mathrm{U}_{1}=\left(\alpha_{\mathrm{s}}-\alpha_{\mathrm{Cu}}\right)\left(\mathrm{T}_{1}-\mathrm{T}_{0}\right) \\
& \mathrm{U}_{2}=\left(\alpha_{\mathrm{s}}-\alpha_{\mathrm{CuNi}}\right)\left(\mathrm{T}_{1}-\mathrm{T}_{0}\right)
\end{aligned}
$$

where $\alpha_{\mathrm{Cu}}$ is the Seebeck coefficient of $\mathrm{Cu}, \alpha_{\mathrm{CuNi}}$ is the Seebeck coefficient of CuNi, and $\alpha_{s}$ is the local Seebeck coefficient of the sample. Combining Eqs. (2) and (3) and eliminating the term $\left(\mathrm{T}_{1}-\mathrm{T}_{0}\right)$, the local Seebeck coefficient of the sample can be obtained as:

$$
\alpha_{\mathrm{s}}=\frac{\mathrm{U}_{1}}{\mathrm{U}_{2}-\mathrm{U}_{1}}\left(\alpha_{\mathrm{Cu}}-\alpha_{\mathrm{CuNi}}\right)+\alpha_{\mathrm{Cu}}
$$

Eq. (4) shows that the local Seebeck coefficient measured by the PSM apparatus is independent of temperature, so that errors due to either temperature control or thermal contact resistance can be ignored. This is the advantage of the PSM.

\subsection{Mathematical models}

Numerical simulations on thermoelectric materials subjected to a heated probe were conducted under the following assumptions.

(1) A two-dimensional model is used to simulate the measurement of the PSM apparatus.

(2) The material properties of thermoelectric sample are isotropic.

(3) The sample is a good thermoelectric material with much lower thermal conductivity than that of the probe, so that obvious temperature variance occurs in the sample.

(4) The probe is heatable and isothermal, allowing the temperature of the probe tip to be constant. 


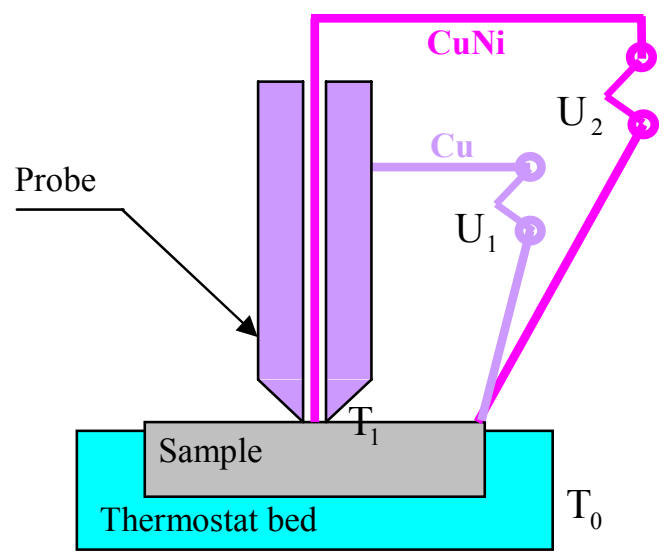

Fig. 2 Schematic representation of the PSM apparatus (reproduced from Ref. 14 and 15)

(5) The probe is assumed to have a negligible influence on the potential distribution in the sample.

(6) The sample-tip contact is perfect, meaning that the contact length equals the probe tip size.

(7) The sample is in good electric and thermal contact with the thermostat bed; the planes of the sample other than the measured plane are thus assumed to be at zero voltage (grounded) and a constant temperature.

(8) The air surrounding the sample is assumed to be insulating and adiabatic.

\subsubsection{Governing equations}

When a heated probe tip is positioned on the surface of the sample for a short time, the transient heat transfer produces a temperature gradient in the sample. The heat conduction equation shown below is employed to determine the temperature distribution in the sample.

$$
\rho c \frac{\partial \mathrm{T}}{\partial \mathrm{t}}+\mathrm{k} \nabla^{2} \mathrm{~T}=0
$$

where $\rho$ is the density, $\mathrm{c}$ is the specific heat, $\mathrm{T}$ is the temperature, $\mathrm{t}$ is the time, and $\mathrm{k}$ is the thermal conductivity. For thermoelectric materials, the temperature gradient induces a thermoelectric voltage. According to the definition of the Seebeck coefficient, the induced open-circuit thermoelectric voltage distribution can be obtained as:

$$
\nabla^{2} \mathrm{~V}=\alpha \nabla^{2} \mathrm{~T}
$$

where $\mathrm{V}$ is the thermoelectric voltage and $\alpha$ is the Seebeck coefficient of the thermoelectric material.

\subsubsection{Initial and boundary conditions}

Because the heating from the probe on the sample surface is for a short time, a transient term appears in the heat conduction equation. At the initial time, the probe does not touch the sample surface; the initial temperature of the sample is thus $\mathrm{T}_{0}$. According to the assumptions mentioned above, the boundary conditions of the sample are illustrated in Fig. 3. 


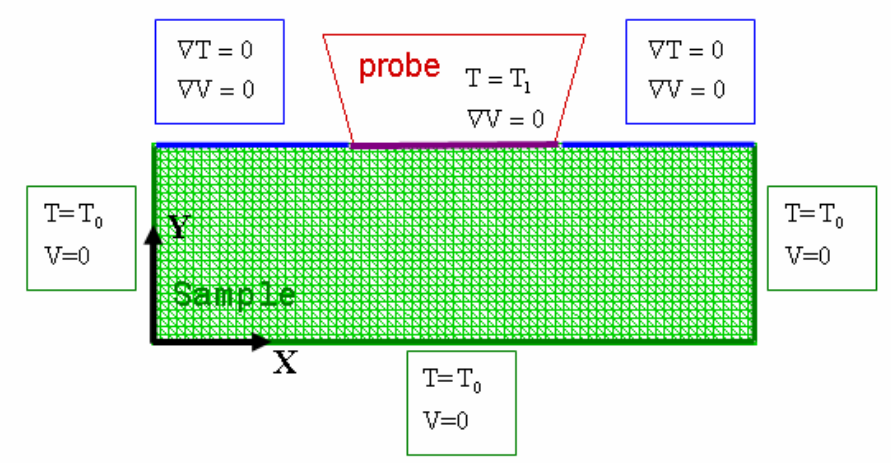

Fig. 3 Boundary conditions of the examined sample.

\subsection{Numerical method}

The Galerkin finite element method is adopted to solve the governing equations shown in Eqs. (5) and (6) and their corresponding boundary conditions. The transient term of Eq. (5) is treated using a fully explicit scheme. Linear triangular elements are used to construct the geometry model of the sample. Note that prior to the simulations, a grid-sensitivity analysis was conducted to determine a suitable mesh size to ensure sufficient accuracy of the simulations results. For a $18 \times 6 \mu^{2}$ sample measured by the $6-\mu \mathrm{m}$ probe tip, 9600 , 15000 , and 21600 structured triangular elements were examined to determine the Seebeck coefficients. The difference between the Seebeck coefficients was small, so 21600 elements were used in this study to obtain a smoother contour.

\subsection{Simulation model}

The local Seebeck coefficient measurements by the PSM apparatus can be simulated using a numerical method via the principle of PSM apparatus. In the simulations, the thermoelectric voltage measured by the probe is the average of the induced thermoelectric voltages of the nodes distributed on the sample-tip contact. According to the principle of the PSM apparatus, $\left(\alpha_{\mathrm{s}}-\alpha_{\mathrm{Cu}}\right)$ and $\left(\alpha_{\mathrm{s}}-\alpha_{\mathrm{CuNi}}\right)$ are individually substituted for $\alpha$ in Eq. (6) to obtain the thermoelectric voltages $U_{1}$ and $U_{2}$, respectively. The local Seebeck coefficient of the sample is then determined using Eq. (4). This model is called the PSM model.

For the practical PSM measurements, the temperature of the probe tip $T_{1}$ and the initial temperature of the sample $\mathrm{T}_{0}$ are kept constant and measured using thermocouples. Two wires are thus used to avoid possible errors. For simulations, $T_{1}$ and $T_{0}$ are used as boundary conditions, so Eq. (2) or (3) can be used to directly calculate the local Seebeck coefficient of the sample (e.g., $\alpha_{\mathrm{s}}=\alpha_{\mathrm{Cu}}+\mathrm{U}_{1} /\left(\mathrm{T}_{1}-\mathrm{T}_{0}\right)$ ). In practice, the metal wires of the PSM apparatus have a nonzero Seebeck coefficient; however, in simulations, the Seebeck coefficient of the metal wire $\alpha_{\text {metal }}$ can be assumed to be zero to obtain the local Seebeck coefficient of the sample more directly (e.g. $\alpha_{\mathrm{s}}=\mathrm{U} /\left(\mathrm{T}_{1}-\mathrm{T}_{0}\right)$, where $\mathrm{U}$ is the simulated thermoelectric voltage measured by the probe when $\left.\alpha_{\text {metal }}=0\right)$ ). Therefore, this simulation model is called the simplified PSM model. The simulation time required for determining the thermoelectric voltages of a given sample for the PSM model is twice that for the simplified PSM model. 


\section{Results and discussion}

A series of simulations of three kinds of thermoelectric material was performed to investigate the local Seebeck coefficient measurements by a heated microprobe via the PSM model and the simplified PSM model. In the simulations, the dimensions of the sample were set to $18 \mu \mathrm{m} \times 6 \mu \mathrm{m}$. The detailed geometrical configurations of the three kinds of thermoelectric material are shown in Fig. 4. Material 1 is a homogeneous thermoelectric material with a Seebeck coefficient of $100 \mu \mathrm{V} / \mathrm{K}$. Material 2 is a two-layer thermoelectric material with Seebeck coefficients of $100 \mu \mathrm{V} / \mathrm{K}$ and $200 \mu \mathrm{V} / \mathrm{K}$. Material 3 is a thermoelectric material with a Seebeck coefficient of $100 \mu \mathrm{V} / \mathrm{K}$ that has an inhomogeneity with a Seebeck coefficient of $200 \mu \mathrm{V} / \mathrm{K}$. Parameters including the probe tip size ( 6 and 1 $\mu \mathrm{m}$ ) and the heating time ( 3 and $2 \mu \mathrm{s}$ ) are investigated for micro scale measurement; the parameters yield a spatial resolution of near $10 \mu \mathrm{m}$. The probe is placed exactly at the center of sample surface for a short time to measure the local Seebeck coefficient. All samples are assumed to have the same material properties everywhere, except for the Seebeck coefficient of the inhomogeneity. The density of the sample is $7740 \mathrm{Kgm}^{-3}$, the specific heat of the sample is $154.4 \mathrm{JK}^{-1} \mathrm{Kg}^{-1}$, the thermal conductivity of the sample is 1.2 $\mathrm{WK}^{-1} \mathrm{~m}^{-1}$, and the electrical resistivity is $9.8 \times 10^{-6} \Omega \mathrm{m}$. The Seebeck coefficients of $\mathrm{Cu}$ and $\mathrm{CuNi}$ are 6.5 and $-40 \mu \mathrm{V} / \mathrm{K}$, respectively. The temperature of the probe is kept at 298 $\mathrm{K}$ and the initial temperature of the sample is $293 \mathrm{~K}$.

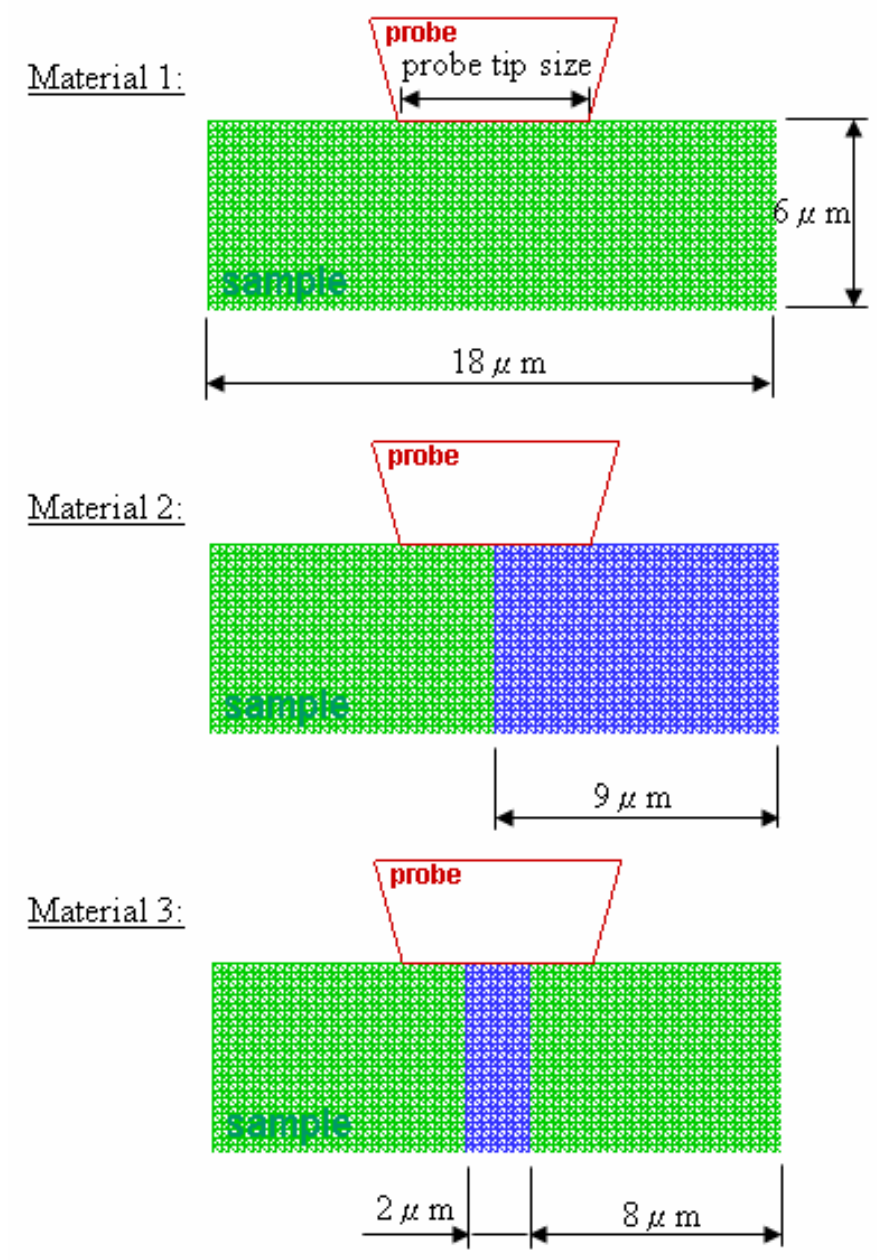

Fig. 4 Schematic of the three kinds of thermoelectric material. The Seebeck coefficient of green parts is $100 \mu \mathrm{V} / \mathrm{K}$ and that of blue parts is $200 \mu \mathrm{V} / \mathrm{K}$. 


\subsection{Simulations of the homogeneous thermoelectric materials}

Simulations for the local Seebeck coefficient measurements on the homogeneous thermoelectric materials (Material 1 in Fig. 4) were conducted. The simulation results indicate that for both the PSM model and the simplified model, all the measured local Seebeck coefficients are equal to the Seebeck coefficients of the homogeneous thermoelectric materials $(100 \mu \mathrm{V} / \mathrm{K})$ for various probe tip sizes $(6$ and $1 \mu \mathrm{m})$ and heating times (3 and $2 \mu \mathrm{s}$ ). The local Seebeck coefficient measured for the homogeneous thermoelectric material is independent of the probe tip size, heating time, and simulation model.

Because the trend of the thermoelectric voltage distribution simulated using the PSM model is similar to that obtained using the in simplified PSM model, the latter was used to study the voltage distribution in subsequent simulations. Fig. 5 shows the simulation results obtained for temperature distribution and voltage distribution in the sample with probe tip sizes of $6 \mu \mathrm{m}$ and heating times of $3 \mu \mathrm{s}$. When the heating time was $3 \mu \mathrm{s}$, a bigger half-circle temperature difference zone appeared in the vicinity of the probe-sample contact area, as shown in Fig. 5 (a). The maximum size of the half-circle temperature difference zone is much bigger than the probe tip size. It can be seen that the induced thermoelectric voltage distribution has a similarity with the temperature distribution in the sample as shown in Fig. 5. The similarity between the induced thermoelectric voltage distribution and the temperature distribution is independent of probe tip size and heating time, as is the case that when the sample is a homogeneous thermoelectric material.

(a)

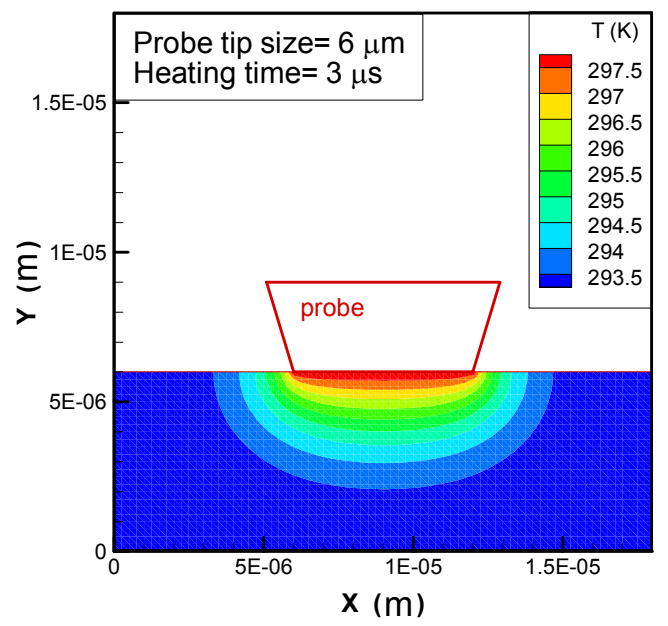

(b)

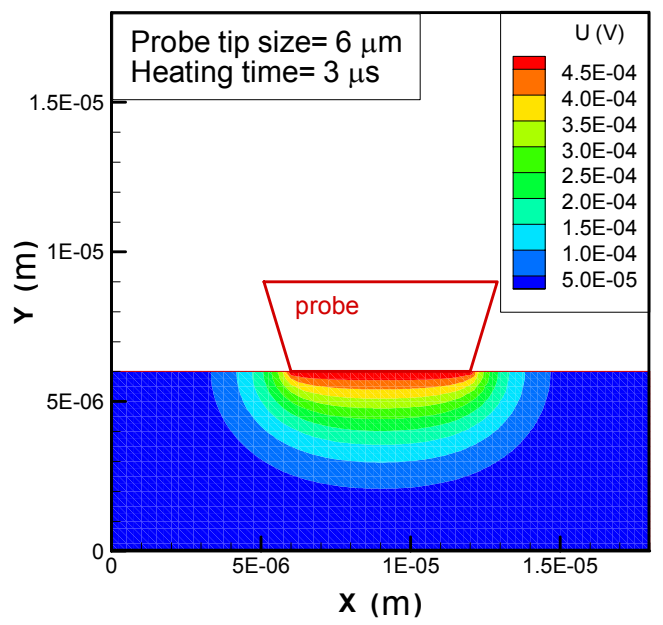

Fig. 5 (a) Temperature (T) contour and (b) voltage (U) distribution of the examined sample in contact with a heated microprobe for a given heating time. Note that the thermal conductivity $(\mathrm{k})$ of the sample is $1.2 \mathrm{WK}^{-1} \mathrm{~m}^{-1}$.

\subsection{Simulations of two-layer thermoelectric material}

Simulations for the local Seebeck coefficient measurements on two-layer thermoelectric materials (Material 2 in Fig. 4) were conducted. The simulation results indicate that for both the PSM model and the simplified model, all the measured local Seebeck coefficients are equal to $150 \mu \mathrm{V} / \mathrm{K}$ for various probe tip sizes $(6$ and $1 \mu \mathrm{m})$ and heating times (3 and $2 \mu \mathrm{s})$. The measured local Seebeck coefficients are all the same $(150 \mu \mathrm{V} / \mathrm{K})$ due to the probe 
position being the same. When the probe was placed on the center of the sample, as shown in Fig. 4, the measured area of each material was a half of the probe area for each measurement. The local Seebeck coefficient measured for the two-layer thermoelectric material is thus independent of the probe tip size, heating time, and simulation model.

Fig. 6 (a) shows the induced thermoelectric voltage distribution of the two-layer sample heated by the probe for a heating time of $3 \mu \mathrm{s}$. It can be seen that the thermoelectric voltage difference zone is distorted and that a higher thermoelectric voltage zone (shown in red) appears in the right part of the central region beneath the probe due to the higher Seebeck coefficient. Fig. 6 (b) shows the thermoelectric voltages distributed on the sample-tip contact of the above two-layer sample for various probe tip sizes (6 and $1 \mu \mathrm{m})$ and heating times ( 3 and $2 \mu \mathrm{s}$ ). It can be seen that the magnitude of thermoelectric voltage profile decreases with increasing heating time; however, each profile remains antisymmetric at a given point. Therefore, the average thermoelectric voltage from each profile remains constant, making the corresponding local Seebeck coefficient constant, which explains why the simulation results are all $150 \mu \mathrm{V} / \mathrm{K}$ in this section.

(a)

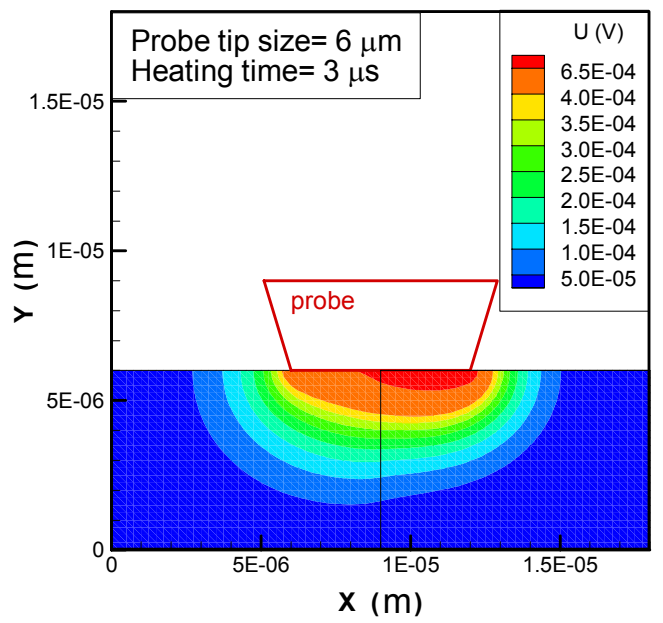

(b)

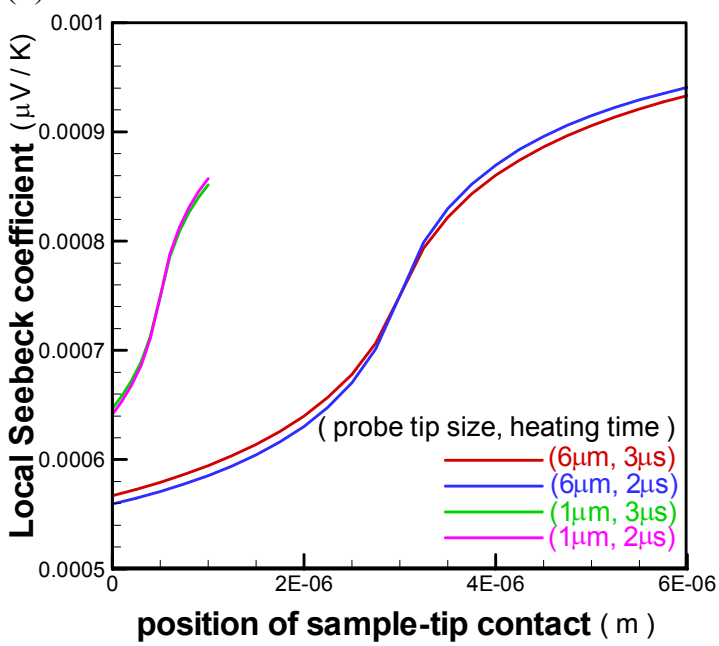

Fig. 6 (a) Voltage (U) distribution of the two-layer sample in contact with a heated microprobe. (b) Comparison of the thermoelectric voltages distributed on the sample-tip contact for various probe tip sizes and heating times.

\subsection{Simulations of thermoelectric material with inhomogeneity}

Table 1 shows the simulation results of the thermoelectric material with an inhomogeneity (Material 3 in Fig. 4) for the local Seebeck coefficient measurements. It can be seen that the measured local Seebeck coefficient depends on the probe tip size and heating time and is independent of the simulation model when the probe is placed on the center of the sample, as shown in Fig. 4. For a given heating time, the local Seebeck coefficient decreases with increasing probe tip size. For a given probe tip size, the local Seebeck coefficient decreases with increasing heating time. 
Table 1 The simulation results of the thermoelectric material with inhomogeneity

\begin{tabular}{|c|c|c|c|c|c|}
\hline Probe tip siz & (unit: $\mu \mathrm{m}$ ) & 6 & 1 & 6 & 1 \\
\hline Heated time & (unit: $\mu s$ ) & 3 & 3 & 2 & 2 \\
\hline $\begin{array}{l}\text { PSM } \\
\text { model }\end{array}$ & $\begin{array}{r}\alpha_{\mathrm{s}}=\frac{\mathrm{U}_{1}}{\mathrm{U}_{2}-\mathrm{U}_{1}}\left(\alpha_{\mathrm{Cu}}-\alpha_{\mathrm{CuNi}}\right)+\alpha_{\mathrm{Cu}} \\
\text { (unit: } \mu \mathrm{V} / \mathrm{K})\end{array}$ & 123.2 & 168.9 & 124.1 & 172.4 \\
\hline $\begin{array}{l}\text { Simplified } \\
\text { PSM model }\end{array}$ & $\alpha_{\mathrm{s}}=\frac{\mathrm{U}}{\left(\mathrm{T}_{1}-\mathrm{T}_{0}\right)} \quad$ (unit: $\left.\mu \mathrm{V} / \mathrm{K}\right)$ & 123.2 & 168.9 & 124.1 & 172.4 \\
\hline
\end{tabular}

(a)

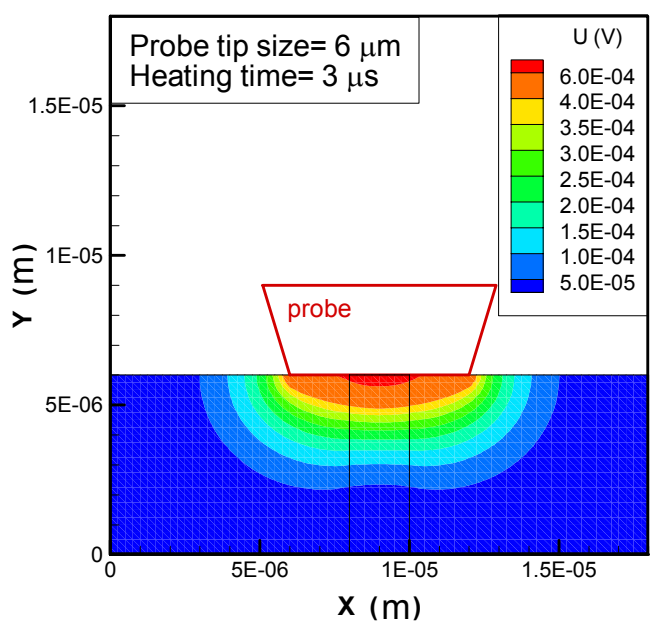

(b)

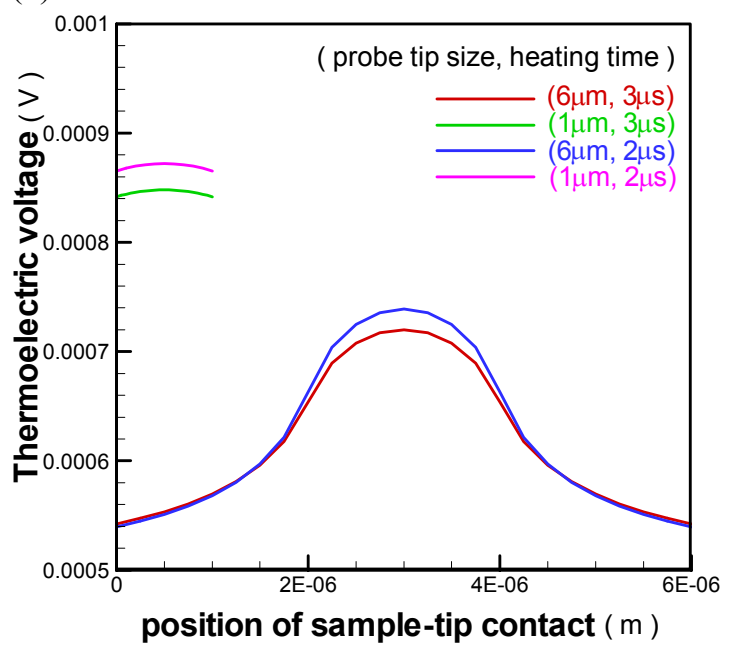

Fig. 7 (a) Voltage (U) distribution of the thermoelectric sample with an inhomogeneity in contact with a heated microprobe. (b) Comparison of the thermoelectric voltages distributed on the sample-tip contact for various probe tip sizes and heating times.

Fig. 7 (a) shows the induced thermoelectric voltage distribution of the thermoelectric sample with an inhomogeneity heated by the probe for $3 \mu \mathrm{s}$. It can be seen that the induced thermoelectric voltage difference zone is distorted and that a higher thermoelectric voltage zone (shown in red) appears in the center region beneath the probe due to the higher Seebeck coefficient of the inhomogeneity. Fig. 7 (b) shows the thermoelectric voltages distributed on the sample-tip contact of the sample with an inhomogeneity for various probe tip sizes $(6$ and $1 \mu \mathrm{m})$ and heating times ( 3 and $2 \mu \mathrm{s})$. It can be seen that the magnitude of the thermoelectric voltage profile decreases with increasing heating time; however, each profile remains symmetric to the central line of the probe. The decrease of the magnitude of the thermoelectric voltage profile with increasing heating time is caused by the extended temperature difference zone. The results indicate that the resolution of PSM apparatus and the accuracy of the Seebeck coefficient of the inhomogeneity increase with decreasing probe tip size and heating time due to the smaller temperature difference zone. In the other words, when the temperature difference zone is small enough to be within the inhomogeneity region, the Seebeck coefficient of the inhomogeneity can be measured. 


\subsection{Study of the spatial resolution of the PSM apparatus}

Fig. 8 shows the simulation results obtained for temperature distribution and voltage distribution in the sample with a probe tip size of $6 \mu \mathrm{m}$ and a heating time of $3 \mu \mathrm{s}$ for a thermal conductivity $(\mathrm{k})$ of the sample of $0.6 \mathrm{WK}^{-1} \mathrm{~m}^{-1}$, which is half of that used in the simulations. Comparing Figs. 5(a) and (b) with Figs. 8 (a) and (b), it can be seen that a sample with higher thermal conductivity has a bigger temperature difference zone and a bigger corresponding thermoelectric voltage difference distribution. Therefore, the magnitude of the temperature difference zone is influenced by the probe tip size, heating time, and the thermal conductivity of the sample.

(a)

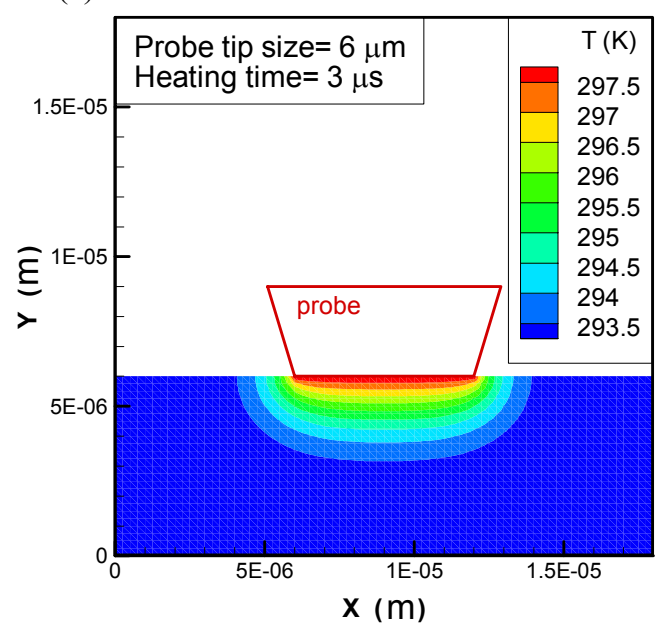

(b)

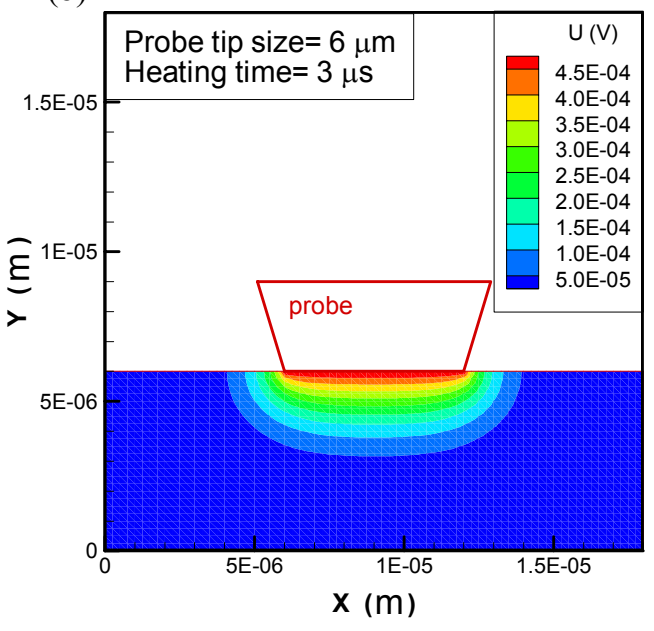

Fig. 8 (a) Temperature contours and (b) voltage distribution of the examined sample in contact with a heated microprobe with a tip size of $6 \mu \mathrm{m}$ for a heating time of $3 \mu \mathrm{s}$. Note that the thermal conductivity $(\mathrm{k})$ of the sample is $0.6 \mathrm{WK}^{-1} \mathrm{~m}^{-1}$.

Based on the results of the previous section, the Seebeck coefficient of the inhomogeneity can be measured when the temperature difference zone is within the inhomogeneity region. By gradually increasing the size of the inhomogeneity in the sample (Material 3 in Fig. 3) to make the temperature difference zone within the inhomogeneity region for the given probe tip size, heating time, and thermal conductivity of the sample, the Seebeck coefficient of the inhomogeneity can be obtained and the resolution of the PSM apparatus can be determined. Fig. 9 (a) shows the variation of thermoelectric voltage distribution on the sample-tip contact with increasing inhomogeneity size. When the thermoelectric voltage profile becomes a straight horizontal line and the corresponding measured local Seebeck coefficient equals the Seebeck coefficient of the inhomogeneity, the corresponding inhomogeneity size is sufficient to make the temperature difference zone within the inhomogeneity region. Fig. 9 (b) shows the measured local Seebeck coefficient of the sample with inhomogeneities of various sizes for various probe tip sizes, heating times, and thermal conductivities of the sample. It can be seen that the measured local Seebeck coefficient increases with increasing inhomogeneity size until the measured local Seebeck coefficient reaches the Seebeck coefficient of the inhomogeneity; the local Seebeck coefficient profile then becomes a straight horizontal line. Fig. 9 (b) also shows that the resolution of the PSM apparatus increases with decreasing probe tip size, heating time, and thermal conductivity of the sample due to the smaller temperature difference zone. Table 2 shows the spatial resolutions for various probe tip sizes, heating times, and thermal 
conductivities of the sample from Fig. 9 (b). In order to extend the spatial resolution determination method to higher thermal conductivities, an inhomogeneity of $10 \mathrm{WK}^{-1} \mathrm{~m}^{-1}$ is applied in the simulation. Fig. 10 shows the measured local Seebeck coefficient of samples with various inhomogeneity sizes for a probe tip size of $6 \mu \mathrm{m}$, a heating time of $3 \mu \mathrm{m}$, and a thermal conductivity of $10 \mathrm{WK}^{-1} \mathrm{~m}^{-1}$. The corresponding spatial resolution is $50 \mu \mathrm{m}$. Therefore, the spatial resolution of the PSM apparatus for local Seebeck coefficient measurement is high when the thermal conductivity of the sample is lower than $1 \mathrm{WK}^{-1} \mathrm{~m}^{-1}$, which agrees with the literature ${ }^{(8)}$.

(a)

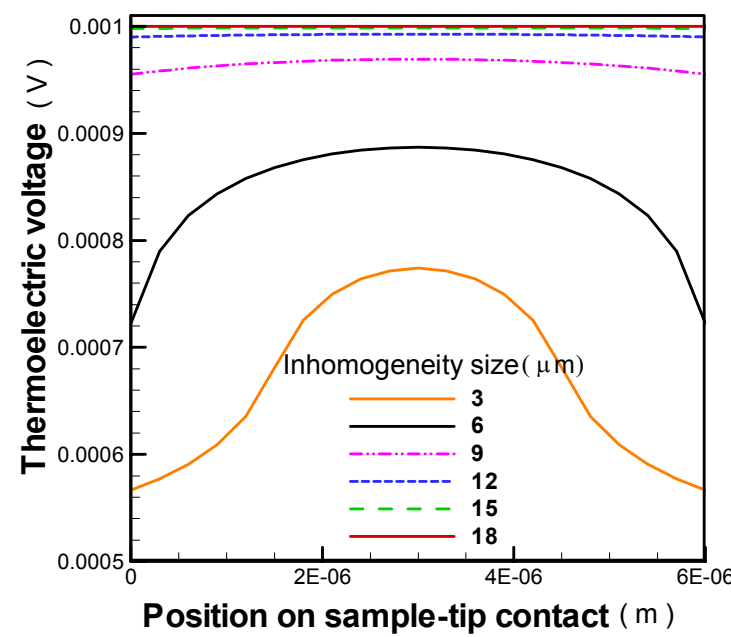

(b)

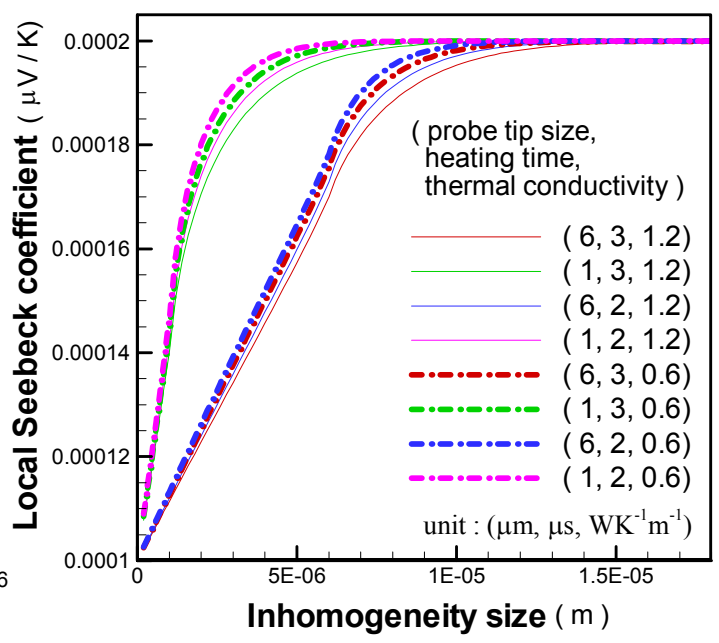

Fig.9 (a) Comparison of the thermoelectric voltages distributed on the sample-tip contact for a probe tip size of $6 \mu \mathrm{m}$ and a heating time of $3 \mu \mathrm{s}$ for thermoelectric samples with inhomogeneities of various sizes. (b) The measured local Seebeck coefficient of samples with various inhomogeneity sizes, probe tip sizes, heating times, and thermal conductivities of the sample.

Table 2 Spatial resolutions for various probe tip sizes, heating times, and thermal conductivities of the sample

\begin{tabular}{|c|c|c|c|c|}
\hline Spatial resolution & $\begin{array}{c}\text { Probe tip size } \\
6 \mu \mathrm{m}\end{array}$ & $\begin{array}{c}\text { Probe tip size } \\
6 \mu \mathrm{m}\end{array}$ & $\begin{array}{c}\text { Probe tip size } \\
1 \mu \mathrm{m}\end{array}$ & $\begin{array}{c}\text { Probe tip size } \\
1 \mu \mathrm{m}\end{array}$ \\
\cline { 2 - 5 } & $\begin{array}{c}\text { Heating time } \\
3 \mu \mathrm{s}\end{array}$ & $\begin{array}{c}\text { Heating time } \\
2 \mu \mathrm{s}\end{array}$ & $\begin{array}{c}\text { Heating time } \\
3 \mu \mathrm{s}\end{array}$ & $\begin{array}{c}\text { Heating time } \\
2 \mu \mathrm{s}\end{array}$ \\
\hline $\begin{array}{c}\text { Thermal conductivity } \\
\mathrm{k}=1.2 \mathrm{WK}^{-1} \mathrm{~m}^{-1}\end{array}$ & $18.0 \mu \mathrm{m}$ & $15.8 \mu \mathrm{m}$ & $13.4 \mu \mathrm{m}$ & $11.2 \mu \mathrm{m}$ \\
\hline $\begin{array}{c}\text { Thermal conductivity } \\
\mathrm{k}=0.6 \mathrm{WK}^{-1} \mathrm{~m}^{-1}\end{array}$ & $14.4 \mu \mathrm{m}$ & $12.8 \mu \mathrm{m}$ & $10.2 \mu \mathrm{m}$ & $9.8 \mu \mathrm{m}$ \\
\hline
\end{tabular}




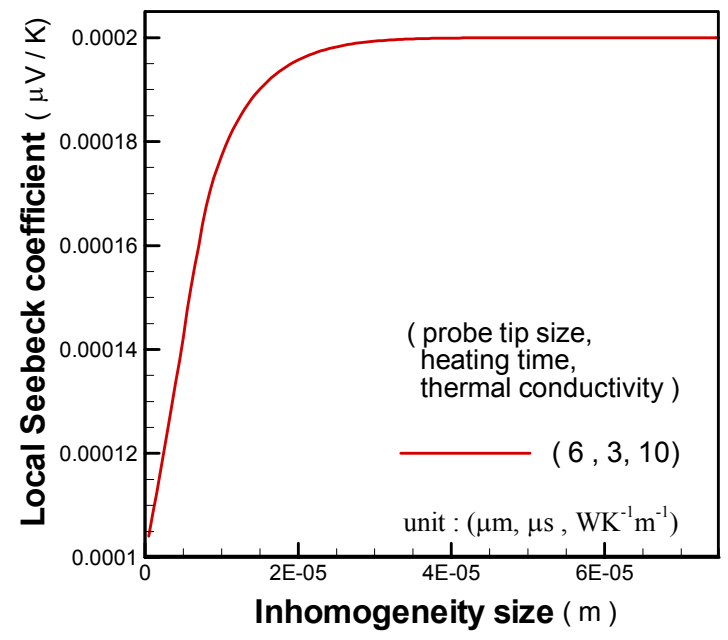

Fig. 10 Measured local Seebeck coefficient of samples with various inhomogeneity sizes for a probe tip size of $6 \mu \mathrm{m}$, a heating time of $3 \mu \mathrm{m}$, and a thermal conductivity of 10 $\mathrm{WK}^{-1} \mathrm{~m}^{-1}$.

\subsection{Study on a film coated on a substrate}

Seebeck coefficient measurements with sufficient spatial resolution may produce a spurious Seebeck coefficient for thermoelectric films coated on a substrate due to the effect of the substrate. This is due to the heating of the probe on the film possibly heating the substrate, creating a temperature difference zone within the film and substrate; therefore, the induced thermoelectric voltage difference zone is within both the film and substrate, making the measured Seebeck coefficient of the film spurious, as shown in Fig. 11 (a) and (b). By reducing the heating time to make the temperature difference zone within only the film, the effect of the substrate may disappear due to the limited heating time and the measurement limitation of the thermoelectric voltage for the PSM apparatus. Before determining the resolution of the PSM apparatus for the thermoelectric film, the effect of the substrate should be verified via simulation to ensure that the measured Seebeck coefficient of the film is not spurious; only then can the above spatial resolution determination method be used to obtain the spatial resolution for the film coated on the substrate to determine the true Seebeck coefficient of the film.

(a)

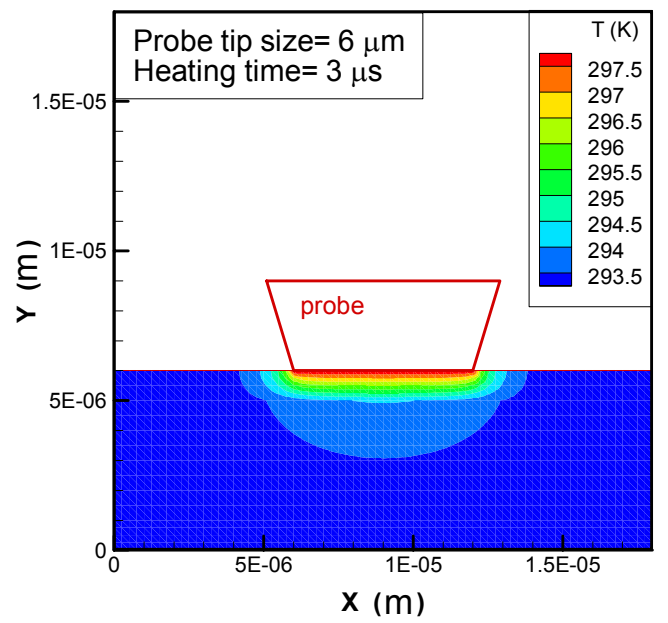

(b)

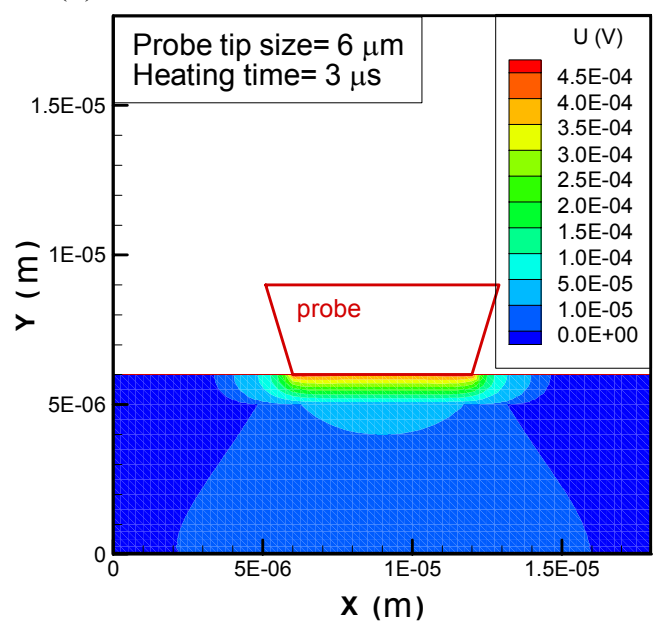


Fig. 11 (a) Temperature (T) contour and (b) voltage (U) distribution of a film coated on a stainless steel substrate. The thermal conductivity of the film is $1.2 \mathrm{WK}^{-1} \mathrm{~m}^{-1}$, the thermal conductivity of the substrate is $14 \mathrm{WK}^{-1} \mathrm{~m}^{-1}$, the Seebeck coefficient of the film is $100 \mu \mathrm{V} / \mathrm{K}$, and the Seebeck coefficient of the substrate is $-1.4 \mu \mathrm{V} / \mathrm{K}$. The thickness of the film is $1 \mu \mathrm{m}$ and that of the substrate is $5 \mu \mathrm{m}$. The measured Seebeck coefficient is $84.3 \mu \mathrm{V} / \mathrm{K}$.

\section{Conclusion}

This paper presented numerical simulations of local Seebeck coefficient measurement for thermoelectric materials by a heated microprobe of the PSM apparatus. The following conclusions were reached:

1. The local Seebeck coefficient measured by the PSM apparatus can be efficiently simulated via the simplified PSM model (i.e., without considering the Seebeck coefficients of wires).

2. A similarity between the temperature difference zone and thermoelectric voltage distribution was found only in the homogeneous thermoelectric material.

3. The distorted voltage distribution of the thermoelectric material with an inhomogeneity is different from the temperature distribution due to the different Seebeck coefficient of the inhomogeneity.

4. On the sample-tip contact area, the proportion of the inhomogeneity affects the distribution of the thermoelectric voltage, leading to a different local Seebeck coefficient.

5. The spatial resolution of the PSM apparatus can be obtained by gradually increasing the size of inhomogeneity in the center part of the thermoelectric material.

6. The magnitude of the temperature difference zone increases and the spatial resolution of the PSM apparatus decreases with increasing probe tip size, heating time, and thermal conductivity of the thermoelectric material.

7. Before determining the spatial resolution of the PSM apparatus for a film coated on a substrate, the measured Seebeck coefficient must be verified to ensure that it is not spurious (i.e., to avoid the effect of the substrate).

\section{References}

(1) Huesgen, T., P. Woias, and N. Kockmann, Design and fabrication of MEMS thermoelectric generators with high temperature efficiency. Sensors and Actuators A: Physical, 2008, 145, pp. 423-429.

(2) Hun Sik, H., et al. Performance measurement and analysis of a thermoelectric power generator. Thermal and Thermomechanical Phenomena in Electronic Systems (ITherm), 2010 12th IEEE Intersociety Conference on, 2010, pp.1-7.

(3) Boukai, A., et al., Silicon nanowires as efficient thermoelectric materials. Nature, 2008. 451(7175), pp. 168-171.

(4) Rowe, D. and V. Shukla, The effect of phonon grain boundary scattering on the lattice thermal conductivity and thermoelectric conversion efficiency of heavily doped fine grained, hot pressed silicon germanium alloy. Journal of Applied Physics, 2009, 52(12), pp. 7421-7426.

(5) Gelbstein, Y., Z. Dashevsky, and M. Dariel, High performance n-type PbTe-based materials for thermoelectric applications. Physica B: Condensed Matter, 2005, 363(1-4), pp. 196-205. 
(6) Chandra, L., et al., Simple and precise thermoelectric power measurement setup for different environments. Review of Scientific Instruments, 2008, 79, pp. 103907.

(7) Kumar, S. and S. Kasiviswanathan, A hot probe setup for the measurement of Seebeck coefficient of thin wires and thin films using integral method. Review of Scientific Instruments, 2009, 79(2), pp. 024302.

(8) Platzek, D., et al. Potential-Seebeck-microprobe (PSM): measuring the spatial resolution of the Seebeck coefficient and the electric potential. Thermoelectrics, 2005. ICT 2005. 24th International Conference on, 2005, pp. 13-16.

(9) Platzek, D., et al. Seebeck Scanning Microprobe for Thermoelectric FGM. Materials Science Forum Vols., 2005, 492-493, pp. 587-592.

(10) Ziolkowski, P., et al. Application Overview of the Potential Seebeck Microscope. ICT '06. 25th International Conference on, 2006, pp. 684-688.

(11) $\mathrm{Wu}, \mathrm{K}$, et al., Improvement of spatial resolution for local Seebeck coefficient measurements by deconvolution algorithm. Review of Scientific Instruments, 2009, 80, pp. 105104.

(12) Rowe, D., Thermoelectrics handbook: Macro to nano. CRC Press, 2006.

(13) Nolas, G., J. Sharp, and H. Goldsmid, Thermoelectrics: basic principles and new materials developments. Springer Verlag, 2001.

(14) Reinshaus, P., et al., Scanning Thermo Probe Technique-a Method for High Resolution Characterization of Graded Semiconductors and Metals. Materials Science Forum vols., 1999, 308-311, pp. 890-895.

(15) Ni, H., et al., Mapping and analysis of microscopic Seebeck coefficient distribution. Journal of Materials Science, 2005. 40(3): pp. 605-608. 